$\xi=\square$

\title{
Fundamental physics and the fine-structure constant
}

\author{
Michael A. Sherbon* \\ Case Western Reserve University Alumnus, United States \\ *Corresponding author E-mail: michael.sherbon@case.edu
}

\begin{abstract}
From the exponential function of Euler's equation to the geometry of a fundamental form, a calculation of the fine-structure constant and its relationship to the proton-electron mass ratio is given. Equations are found for the fundamental constants of the four forces of nature: electromagnetism, the weak force, the strong force and the force of gravitation. Symmetry principles are then associated with traditional physical measures.
\end{abstract}

Keywords: Euler's Equation; Fine-Structure Constant; Forces of Nature; Fundamental Constants; Symmetry Principles.

\section{Introduction}

Leonhard Euler was one of the greatest mathematicians of the eighteenth century. As a mathematician Lokenath Debnath states in The Legacy of Leonhard Euler, "It is remarkable that Euler discovered two elegant and most beautiful formulas in mathematics." [1].

$\mathrm{e}^{\mathrm{i} \pi}+1=0 \quad$ and $\quad \mathrm{e}^{2 \pi \mathrm{i}}-1=0$.

William Eisen's description and interpretation of Euler's equation $\mathrm{e}^{\mathrm{i} \pi}+1=0$ in relation to the Great Pyramid design shows four curves of $\mathrm{e}^{\mathrm{x}}$ from $\mathrm{x}=0$ to $\mathrm{x}=\pi$, one curve on each side. Dividing the sides by $\pi$ lengths results in a small square in the center called the Golden Apex A, the geometry and symmetry thought to generate the four fundamental forces of nature [2]. Eisen then asks the obvious question about the exponential function and Golden Apex interpretation, "Just how could the builders of the Great Pyramid have been so knowledgeable of the mathematics of the universe ...?"

$\mathrm{A}=\mathrm{e}^{\pi}-7 \pi-1 \simeq \sqrt{ }(2) / 3 \pi \simeq 0.1495$.

$\mathrm{A}$ is the side of the Golden Apex square. $\sqrt{ }(\mathrm{A}) \simeq \mathrm{e} / 7$ and $\mathrm{A}+1=\mathrm{e}^{\pi}-7 \pi \simeq \mathrm{R} \simeq 1.152$, radius of the regular heptagon with side one. The $\sin (2 \pi A) \simeq \varphi / 2$, where $\varphi$ is the golden ratio, $1 / 2 \varphi$ $\simeq \varphi \sqrt{ }(\mathrm{A})$. The $\tan (2 \pi \mathrm{A}) \simeq 1+\sqrt{ }(\mathrm{A}) \simeq \mathrm{K} / 2 \pi$, see Eq. (7) [3]. The polygon circumscribing constant is $K \simeq 2 \tan (3 \pi / 7) \simeq \varphi^{2} / 2 \mathrm{~A}$, see Eq. (7) and discussion [3]. A is also the reciprocal harmonic of the gravitational constant. Also, $\mathrm{A} \simeq \tan ^{2} \mathrm{e}^{-1}$ and $\mathrm{A}^{-1} \simeq \cosh ^{2}(\sqrt{ }(7 / \mathrm{e}))$ $\simeq \varphi \sqrt{ }(2 \pi \mathrm{e}) . \ln \left(\mathrm{A}^{-1}\right) \simeq \pi / \sqrt{ }(\mathrm{e}) \simeq 6 / \pi$, with the cube-sphere proportion. The regular heptagon radius, $\mathrm{R}=\csc (\pi / 7) / 2 \simeq \varphi / \sqrt{ }(2) \simeq$ $\cot ^{2} \alpha^{-1}$ and $2 \pi \mathrm{R} \simeq 1 / \varphi \sqrt{ }(\alpha)$. Also, $\mathrm{RA} \simeq 2 \sqrt{(}(\alpha)$, where alpha $\alpha$ is the fine-structure constant, see the Eq. (7) discussion.

$\mathrm{RA} \simeq \sqrt{ }(\varphi) / \mathrm{e}^{2} \simeq \ln (\pi / \sqrt{ }(7)) \simeq \sqrt{ }(7 / \pi) / \mathrm{K}$.

$\mathrm{R}^{-1} \simeq \sqrt{ }(\varphi) \sin \alpha^{-1}$ with $\mathrm{e}^{\pi}-\pi^{\mathrm{e}} \simeq \sin \alpha^{-1}$ [3]. The $\cosh ^{2}(\sqrt{ }(\mathrm{A})) \simeq$ $\mathrm{e}^{\mathrm{A}}-\mathrm{A}^{\mathrm{e}} \simeq \pi / \mathrm{e}$. The silver constant from the heptagon is $\mathrm{S} \simeq \sqrt{ }$ $(\pi / 2 \mathrm{~A}) \simeq 2 \sqrt{ }(2) \mathrm{R} \simeq 3.247 . \sqrt{ }(\mathrm{S})=2 \cos (\pi / 7) \simeq 7 \varphi / 2 \pi, 2 \pi \mathrm{A} \simeq$ $\mathrm{S} / 2 \sqrt{ }(3)$ and $\mathrm{S} / \sqrt{ }(\pi) \simeq \sqrt{ }(11 / \mathrm{S}) \simeq \sqrt{ }(1 / 2 \mathrm{~A}) \simeq \ln (2 \pi)$. Again, Golden Apex A:

$\mathrm{A} \simeq \sqrt{ }(11) / 7 \pi \simeq \sqrt{ }(\mathrm{e}) / 11 \simeq \sqrt{ }(\pi \alpha) \simeq 2 \pi \alpha \mathrm{S}$.

With the fine-structure constant, $2 \pi \alpha$ is equal to the electron Compton wavelength divided by the Bohr radius and $\pi \alpha$ is the percentage of light absorbed by graphene [4]. $4 / \pi \simeq \sqrt{ }(\mathrm{A}) / 2 \mathrm{~A} \simeq$

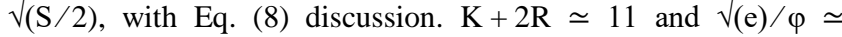
$1+\alpha \varphi^{2}[3]$.

\section{The nature of the fine-structure constant}

Introduced by Arnold Sommerfeld, the fine-structure constant determines the strength of the electromagnetic interaction. Alpha, the fine-structure constant is $\alpha=\mathrm{e}^{2} / \hbar \mathrm{c}$ in cgs units [3]. The finestructure constant related to the Golden Apex of the Great Pyramid:

$2 \mathrm{~A} \simeq 2 \sqrt{ }(\pi \alpha) \simeq 4 \mathrm{~m}_{\mathrm{e}} / \mathrm{m}_{\mathrm{p}} \simeq \simeq \varphi^{2} / \mathrm{K}$

Also, $2 \mathrm{~A} \simeq \tanh \mathrm{S}^{-1} \simeq \tan ^{2}(1 / 2) \simeq \sqrt{ }(\mathrm{K}) / \pi^{2}$ and $\sqrt{ }(2 \mathrm{~A}) \simeq \sqrt{ }(\pi) / \mathrm{S}$. When substituting the fine-structure constant value and approximate value for the proton-electron mass ratio $\alpha \mathrm{m}_{\mathrm{p}} / 4 \mathrm{~m}_{\mathrm{e}} \simeq \varphi+\sqrt{ }(3)$ and $\ln \left(\mathrm{m}_{\mathrm{p}} / \mathrm{m}_{\mathrm{e}}\right) / \ln \left(\alpha^{-1}\right) \simeq 2 \pi \varphi \mathrm{A}$, see the discussion of Eq. (14) [3].

The Wilbraham-Gibbs constant is $\mathrm{G}_{\mathrm{w}}$ and the sinc function is the $\operatorname{sinc} x=\sin \mathrm{x} / \mathrm{x}[5]$ :

$\mathrm{G}_{\mathrm{w}}=\int_{0}^{\pi} \sin \mathrm{c} \mathrm{xdx} \simeq \mathrm{e} \sin \alpha^{-1} \simeq \mathrm{K} / \sqrt{ }(7 \pi)$.

The Wilbraham-Gibbs constant $\mathrm{G}_{\mathrm{w}} \simeq \varphi \ln \pi \simeq \varphi^{2} / \sqrt{ }(2) \simeq 1.852$. The Wilbraham-Gibbs constant is related to the overshoot of Fourier sums in the Gibbs phenomena [5] and other approximations: $\mathrm{G}_{\mathrm{w}} \simeq \sec (1) \simeq \exp \left(\varphi^{-1}\right) \simeq 5 / 7 \sqrt{ }(\mathrm{A})$, see the discussion of Eq. (17).

The inverse Kepler-Bouwkamp constant is the polygon circumscribing constant $\mathrm{K}[3]$ : 
$\mathrm{K}=(\pi / 2) \prod_{n=1}^{\infty} \operatorname{sinc}((2 \pi) /(2 \mathrm{n}+1))=\prod_{n=3}^{\infty} \sec ((\pi) /(n))$.

Also, the polygon circumscribing constant $\mathrm{K} \simeq 2 \pi(1+\sqrt{ }(\mathrm{A})) \simeq$ $\sqrt{ }(7 / 4 \pi \alpha) \simeq \sqrt{ }(11) \varphi^{2}$ and $\mathrm{K} \simeq \varphi^{2} / 2 \mathrm{~A} \simeq 5 \mathrm{~A} / \sqrt{ }(\alpha) \simeq 4 \pi \sqrt{ }(\mathrm{SA}) \simeq$ $3 / 2 \mathrm{RA} \simeq 2 \tan (3 \pi / 7) \simeq 8.7 \mathrm{KA} \simeq \sqrt{ }(\mathrm{e} / \varphi) \simeq \mathrm{R}+\mathrm{A}$.

Half the face apex angle of the Great Pyramid plus half the apex angle is approximately $70^{\circ}$ and $\sin \alpha^{-1} \simeq 2 \cos 70^{\circ}$ [3]. The $\csc \alpha^{-1}$

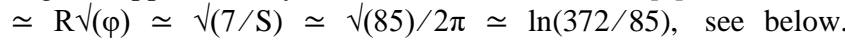
$528 / 504 \simeq 7 \mathrm{~A}$, see discussion of Eq. (17) [3]. First level sum of Teleois proportions is 85 , foundational in Great Pyramid design [3]. $85 / 11 \simeq \mathrm{R} / \mathrm{A}$ and $528 / 85 \simeq 2 \pi$, see Eq. (17). The latest value for the inverse fine-structure constant by Aoyama et al, $\alpha^{-1} \simeq$ 137.035999157 (41), from the most recent experimental results and quantum electrodynamics [6]. Eq. (8) derives the approximate value for $\alpha^{-1} \simeq 137.035999168$ [7]:

$\sin \alpha^{-1} \simeq 504 / 85 \mathrm{~K}=7 ! /(713+137) \mathrm{K}$.

The sum of the eight main resonant nodes on the Turenne Rule is $372 \simeq \mathrm{e} / \alpha$, part of a spectrum analysis that is also related to crystallographic groups [8]. $\mathrm{K} \simeq \varphi \ln (372-137), \ln 372 \simeq 1+\ln 137 \simeq$ $\sqrt{ }(\pi) / 2 \mathrm{~A}$ and $\ln (137 \times 372) \simeq \varphi / \mathrm{A} .137^{2}+372^{2} \simeq 396^{2}$ and from the harmonic radii of the Cosmological Circle, $108+396=504$ [3]. $504 / 396=14 / 11 \simeq 4 / \pi$. Pyramid base angle $\theta_{\mathrm{B}} \simeq \tan ^{-1}(4 / \pi)$. The $\ln (4 / \pi) \simeq \mathrm{A} \varphi$ and $\pi / 4 \simeq \csc \alpha^{-1}-\sin \alpha^{-1} \simeq \sin \theta_{\mathrm{B}}$. The pyramid apex angle $\theta_{\mathrm{A}}, \sin \alpha^{-1} \simeq \theta_{\mathrm{B}} / \theta_{\mathrm{A}} \simeq \sqrt{ }(2) \tan (\pi / 7) \simeq$ $\sqrt{ }(\mathrm{S} / 7) \simeq 2 \pi / \sqrt{ }(85)[3] .528 / 396=4 / 3$ and $396 / 85 \simeq \sqrt{ }(\mathrm{S} / \mathrm{A})$. The quartz crystal harmonic is $\mathrm{Q}_{\mathrm{c}}=786432$ and $1 / \sqrt{ }(\alpha) \simeq \ln \left(\mathrm{AQ}_{\mathrm{c}}\right)$. Base octave harmonic of $Q_{c}, 192 \simeq 7 \pi K$ and $\varphi / \pi \simeq 192 / 372$ [7].

\section{The four fundamental forces of nature}

The heptagon is a feature of the Cosmological Circle, geometric template for many ancient architectural designs; related to the cycloid curve and history of the least action principle [7]. Golden Apex A, silver constant S and fine-structure constant, see Eq. (4).

$\alpha_{\mathrm{E}} \simeq \exp (-2 / \mathrm{Ae}) \simeq \mathrm{A} / 2 \pi \mathrm{S} \simeq 7.29 \times 10^{-3}$.

The electromagnetic coupling constant is $\alpha_{\mathrm{E}}=\alpha=\mathrm{e}^{2} / \hbar \mathrm{c}$ in cgs units [7]. Together with Eq. (12) the ratio $\alpha_{\mathrm{W}} / \alpha_{\mathrm{E}} \simeq \mathrm{A} \pi^{3} \simeq \sqrt{ }(\pi) \varphi^{2}$. $\sqrt{ }(\alpha) \simeq \mathrm{Ae} / \varphi \sqrt{ }(\mathrm{K}) \simeq \sqrt{ }(\mathrm{R}) / 4 \pi$ and $2 \pi \varphi \mathrm{R} \simeq 1 / \sqrt{ }(\alpha) . \mathrm{G}=\hbar \mathrm{c} / \mathrm{mP}^{2}$ $\simeq 6.67191(99) \times 10^{-11} \mathrm{~m}^{3} \mathrm{~kg}^{-1} \mathrm{~s}^{-2}$ is the gravitational constant with Planck mass $\mathrm{mP}_{\mathrm{P}}[9,10]$. Gravitational coupling is $\alpha_{\mathrm{G}}=\mathrm{Gm}_{\mathrm{e}}^{2} / \hbar \mathrm{c}=\left(\mathrm{m}_{\mathrm{e}} / \mathrm{m}_{\mathrm{P}}\right)^{2}$, with Golden Apex A:

$\alpha_{\mathrm{G}} \simeq \exp (-\mathrm{K} \sqrt{ }(\pi) / \mathrm{A}) \simeq 1.752 \times 10^{-45}$.

Also, $-\ln \left(\alpha_{\mathrm{G}}\right) \simeq 2 \mathrm{R} / \mathrm{A}^{2} \simeq \exp \left(\mathrm{A} \pi^{3}\right) \simeq 2 \pi \mathrm{R}_{\mathrm{d}} / \sqrt{ }(\alpha)$, where dodecahedron circumradius $R_{d}=\varphi \sqrt{ }(3) / 2$. $A \pi^{3} \simeq$ Ksin $\theta_{C}$, where $\theta_{C} \simeq$ $32^{\circ}$, half the Great Pyramid face apex angle.

An approximation with the Golden Apex A for the strong force coupling constant [11]:

$\alpha_{\mathrm{S}} \simeq \exp (\mathrm{A}-\sqrt{ }(2) \varphi) \simeq \mathrm{A} / \sqrt{ }(\varphi) \simeq 1.177 \times 10^{-1}$.

The tetrahedral angle $\theta_{\mathrm{t}} \simeq 109.5^{\circ}, \csc ^{2}\left(\theta_{\mathrm{t}}\right)=9 / 8$, the whole tone. $\alpha_{\mathrm{S}} \simeq \ln (9 / 8) \simeq \sqrt{ }(\alpha) \tan (2 \pi \mathrm{A}) \simeq \sqrt{ }(2 / \mathrm{A}) / \pi^{3} \simeq \mathrm{K} \sqrt{ }(\alpha) / 2 \pi$, $\sqrt{ }(2) \varphi-\mathrm{A} \simeq \mathrm{e} / \sqrt{ }(\varphi)$ and $\alpha \mathrm{s}^{-1} \simeq 7 \pi \sqrt{ }(\mathrm{A}) \simeq \pi^{2} / \mathrm{R}[7]$.

Approximation with the Golden Apex for the coupling constant of the weak force [12]:

$\alpha_{\mathrm{W}} \simeq \exp (-1 / 2 \mathrm{~A}) \simeq 2 \mathrm{~A} / \mathrm{K} \simeq 3.4 \times 10^{-2}$.

Also, $\alpha_{\mathrm{W}} / \alpha_{\mathrm{E}} \simeq \mathrm{A} \pi^{3} \simeq \sqrt{ }(\pi) \varphi^{2}$. Coupling constant $\alpha_{\mathrm{W}}=\mathrm{g}_{\mathrm{w}}{ }^{2} / 4 \pi \simeq$ A $/ \sqrt{ }(2) \pi$, where $g_{w}$ is the coupling constant mediating the weak interaction. The Fermi coupling constant $\mathrm{G}_{\mathrm{F}}$ determines the strength of Fermi's interaction that explains the beta decay caused by the weak nuclear force. $G_{F}=g_{w}{ }^{2} / 4 \sqrt{ }(2) m_{w}{ }^{2}=\alpha w \pi / \sqrt{ }(2) m_{w}$ [13], $\mathrm{m}_{\mathrm{w}}$ is the mass of the $\mathrm{W}$ boson. The weak interaction is me- diated by the exchange of $\mathrm{W}$ and $\mathrm{Z}$ gauge bosons. Weinberg angle $\theta_{\mathrm{W}}, \sin ^{2} \theta_{\mathrm{W}} \simeq \sqrt{ }(\alpha) \varphi^{2} \simeq 3 \mathrm{~A} / 2$ [12], [14], Eq. (17); $\cos \theta_{\mathrm{W}}=\mathrm{mw}_{\mathrm{W}} / \mathrm{m}_{\mathrm{Z}}$ $\simeq \sqrt{ }(2) / \varphi \cdot \mathrm{K}^{2} \simeq \cos \left(2 \theta_{\mathrm{w}}\right) / \alpha \simeq \mathrm{A} \sqrt{ }(6 / \pi)$. The pyramid base angle $\theta_{\mathrm{B}}, \theta_{\mathrm{W}} / \theta_{\mathrm{B}} \simeq \sqrt{ }(2 \mathrm{~A}) \simeq \sqrt{ }(\pi) / \mathrm{S}$.

\section{Symmetry principles and physical measures}

Steven Weinberg is often quoted, “... there are symmetry principles that dictate the very existence of all the known forces of nature." [15]. Saul-Paul Sirag says, "By far the deepest theoretical advance afforded by the group-theory approach is the set of ADE Coxeter graphs. It is plausible to think of the ADE graphs as the ultimate Platonic archetypes." [16]. the discovery of symmetry principles and their application [17], [18] has advanced to the monster group and the modular $\mathrm{j}$-function (referred to as "monstrous moonshine") [19], the umbral moonshines and string theory; along with vertex operator algebras in related physical approaches with conformal field theory [20]. For a half-period ratio of $\tau$, the modular function $\mathrm{j}(\tau)$ with $\mathrm{q}=\exp (2 \pi \mathrm{i} \tau)$ has the Fourier expansion [21]:

$J(\tau)=q^{-1}+744+196884 q+21493760 q^{2}+\ldots$

The $\ln (21493760 / 196884) \simeq \sqrt{ }(7 \pi) \simeq \sqrt{ }(2) / 2 \mathrm{~A}$. The $\ln 196884 \simeq$ $\sqrt{ }(2 / \mathrm{A}) / 2 \mathrm{~A} \simeq \mathrm{KR}_{\mathrm{d}}$ and $\mathrm{R}_{\mathrm{d}}=\varphi \sqrt{ }(3) / 2$ is the circumradius of the regular dodecahedron with the side equal to one. From Grumiller et al, the chiral half of the monster group conformal field theory originally proposed by Ed Witten has a partition function given by the j-function. "The number 196884 is interpreted as one Virasoro descendant of the vacuum plus 196883 primary fields corresponding to flat space cosmology horizon microstates." The quantum correction in the respective low-energy entropy is then proportional to the $\ln 196883$ [22].

G.f. is a Fourier series which is the convolution square root of $\mathrm{j}(\tau)$, see Eq. (13) [23].

G.f. $=q^{-1}+372 q+29250 q^{3}-134120 q^{5}+\ldots$.

The $\ln (29250 / 372) \simeq \varphi^{2} / 4 \mathrm{~A} \simeq \mathrm{K} / 2$ and the $\ln (2 \times 372) \simeq \mathrm{A}^{-1}$. The $\ln 372 \simeq \varphi \sqrt{ }(2 / \mathrm{A}) \simeq \sqrt{ }(\pi) / 2 \mathrm{~A} \simeq 2 \mathrm{~K} \cos 70^{\circ}$ and $\sin 70^{\circ} \simeq$ $372 / 396 \simeq 2 \pi \mathrm{A}$, with the hieroglyphic geometry for gold. An application in a special case of umbral moonshine is the Mathieu moonshine work of Eguchi, Ooguri and Tachikawa [24] and followed by others, includes the q-series e(q) whose coefficients are then "... twice the dimension of some irreducible representation of the Mathieu group $\mathrm{M}_{24}$." Pierre-Philippe Dechant continues, "Modularity is therefore very topical, also in other areas and a Clifford perspective on holomorphic and modular functions could have profound consequences." [25]. A normalized q-series e (q):

$e(q)=90 q+462 q^{2}+1540 q^{3}+4554 q^{4}+11592 q^{5}+\ldots$.

The $\ln (462 / 90) \simeq \sqrt{ }(7) / \varphi \simeq \sqrt{ }(\mathrm{A}) \varphi^{3}$ with the $\ln 90 \simeq \sqrt{ }(3) \varphi^{2} \simeq$ $2 / 3 \mathrm{~A} \simeq \mathrm{U} \sqrt{ }(\mathrm{K})$, see Eq. (16). Also correlated, $90 / 372 \simeq \varphi \mathrm{A}$ and $462 / 372 \simeq \varphi \mathrm{A}+1 \simeq 2 / \varphi$, recall discussion of Eq. (8).

A gold pyramid at the tip of the Great Pyramid, with octahedral and icosahedral symmetry [26], was the "Golden Tip" described by John Michell with the support of Algernon Berriman's metrology [27]. This was represented by the height of a pyramid with 5 cubic inches, $0.152 \simeq 11.7 /(7 \times 11), \sqrt{ }(137) \simeq 11.7$ and 0.152 is the tenth part of the Greek cubit of 1.52'. This pyramidion might have been similar to the legendary Golden Sun Disc of Mu. Apex angle of the regular heptagon triangle is $3 \pi / 7$ and an approximation to the apex angle of the Great Pyramid [7]. The "Golden Tip" harmonic of $\mathrm{U} \simeq \varphi \sin 70^{\circ} \simeq 2 \pi \varphi \mathrm{A} \simeq \ln \left(\mathrm{m}_{\mathrm{p}} / \mathrm{m}_{\mathrm{e}}\right) / \ln \left(\alpha^{-1}\right) \simeq 1.52$ [3]. From the heptagon geometry found in the Cosmological Circle, $\mathrm{AU} \simeq \mathrm{R} / \pi \varphi \simeq 5 / 7 \pi \simeq \cot (3 \pi / 7) \simeq 0.227$ and the $\tan (3 \pi / 7)$ $\simeq \mathrm{e} \varphi \simeq 4.4$. The ratio $7 / 5 \simeq \varphi / \mathrm{R} \simeq \mathrm{R}_{\mathrm{d}}$, the circumradius of the regular dodecahedron having the side equal to one, see the discussion following Eq. (13) and [7]. 
$\mathrm{AU} \simeq 85 / 372 \simeq \varphi \sqrt{ }(\mathrm{e} \alpha) \simeq \exp (-\mathrm{ARK}) \simeq 2 / 3 \sqrt{ }(\mathrm{K})$.

The sum $\mathrm{A}+\mathrm{U} \simeq 1 / 4 \mathrm{~A}, \mathrm{~A} / \mathrm{U} \simeq \mathrm{R} \sqrt{ }(\alpha)$ and the $\mathrm{ARK} \simeq \sqrt{ }(7 / \pi) \simeq$ $\operatorname{coth}^{2} \mathrm{R} \simeq 3 / 2 \simeq 10 \mathrm{~A}$.

Interesting parallels can be found between William Eisen's Golden Apex of the Great Pyramid design, the torus topology of Einstein's relativity and Wolfgang Pauli's World Clock Vision; with his i ring imaginal geometry of the unit circle on the complex plane and also symbolic of the unus mundus as the natural ontology of quantum theory [28].

Pauli's World Clock also has the golden ratio geometry related to the fine-structure constant together with four men swinging pendulums [28]. Ancient Egyptian architects converted celestial time periods into lengths that are equivalent to those of the pendulum measures as rediscovered by Galileo and explained by Sir Isaac Newton. Roger Newton states that "speculations concerning a long-awaited reconciliation between Einstein's general theory of relativity and the quantum, known in their various guises as superstring theory, employ as their basic element the properties of Galileo's simple pendulum." [29]. Flinders Petrie found the harmonic of the standard day when converted by the pendulum formula results in the length of the Royal Cubit, "... basis of the Egyptian land measures .... This value for the cubit is 20.617 " while the best examples in stone are $20.620 " \pm 0.005$ "." The Egyptian Royal Cubit is the traditionally known measure basis of the Great Pyramid “... and its base measures 440 Royal Cubits in length." [30].

R.C. $\simeq 144 / 7 \simeq \mathrm{A} / \alpha \simeq \varphi+\varphi / \sqrt{ }(\alpha) \simeq \sqrt{ }(7 \pi) / \mathrm{AU} \simeq 7 \sqrt{ }(\mathrm{K})$.

The canonical Royal Cubit of 20.736" is the harmonic of $144^{2}$ and the standard harmonic Royal Cubit R.C. $\simeq 20.618$ [27]. Also, R.C. $\simeq \sqrt{ }(7 \pi) \tan (3 \pi / 7)$. Basic square perimeter of the Cosmological Circle is 44 and $44^{2}+137^{2} \simeq 144^{2}[28] .22=\sqrt{ }(44+440) \simeq 7 \pi$ and $\mathrm{A} \simeq \pi / \sqrt{ }(440)[3] .372 / 440 \simeq 4 \sqrt{ }(2) \mathrm{A}, 144 / 372 \simeq \sqrt{ }(\mathrm{A})$ and $504 / 144=7 / 2$. Also, $144 / 85 \simeq \mathrm{e} / \varphi \simeq \mathrm{A}+\mathrm{U}$ [3]. Plato's "fusion number" 1746 , described by John Michell, represented the apex of the Great Pyramid [27]. Fusion number $1746 \simeq 144 \sqrt{ }(7 \pi / \mathrm{A})$, $372 / 1746 \simeq \sqrt{ }(2) \mathrm{A}$ and $\mathrm{AU} \simeq 396 / 1746.528 / 372 \simeq \sqrt{ }(2)$, $528 / 1746 \simeq 2 \mathrm{~A}, 528 / 504 \simeq 7 \mathrm{~A}, 1746 / 504 \simeq 2 \sqrt{ }(3)$ and R.C. $\simeq$ $1746 / 85$. The Great Pyramid Key is $528 \simeq \ln (7 / \mathrm{A}) / \alpha[3]$.

\section{Conclusion}

From the exponential function of Euler's equation to the geometry of a fundamental form, the Golden Apex of the Great Pyramid was described, leading to a calculation of the fine-structure constant and its close relationship with the proton-electron mass ratio. Golden Apex related equations were then found for four fundamental forces of nature.

Juan Maldacena restates it, "The forces of nature are based on symmetry principles." [31]. these symmetry principles were then associated with traditional physical measures. And finally, here is William Eisen's quotation again, "Just how could the builders of the Great Pyramid have been so knowledgeable of the mathematics of the universe ...?" [2].

\section{Acknowledgement}

Special thanks to Case Western Reserve University, MathWorld and WolframAlpha

\section{References}

[1] L. Debnath, The Legacy of Leonhard Euler: A Tricentennial Tribute, Imperial College Press, London, World Scientific Publishing, River Edge, NJ, (2009) p.180. https://doi.org/10.1142/p698.

[2] W. Eisen, The Essence of the Cabalah, DeVorss, Marina Del Rey, CA, 1984, pp.474-479.

[3] M.A. Sherbon, Quintessential nature of the fine-structure constant, Global Journal of Science Frontier Research A, 15, 4 (2015) 23-26.
[4] R.R. Nair, et al, Fine structure constant defines visual transparency of graphene, Science, 320, $5881 \quad$ (2008) 1308-1308. https://doi.org/10.1126/science.1156965.

[5] Z. Zi-Xiang, An observation of relationship between the fine structure constant and the Gibbs phenomenon in Fourier analysis, Chinese Physics Letters, $21.2 \quad$ (2004) 237-238. https://doi.org/10.1088/0256-307X/21/2/006.

[6] T. Aoyama, M. Hayakawa, T. Kinoshita, \& M. Nio, Tenth-order electron anomalous magnetic moment: contribution of diagrams without closed lepton loops, Physical Review D, 91, 3 (2015) 033006.

[7] M.A. Sherbon, Fundamental nature of the fine-structure constant, International Journal of Physical Research, 2, 1 (2014) 1-9. https://doi.org/10.14419/ijpr.v2i1.1817.

[8] C.B. Hills, Supersensonics, University of the Trees, Boulder Creek, CA, 1978, p.120.

[9] G. Rosi, et al, Precision measurement of the Newtonian gravitational constant using cold atoms, Nature, 510.7506 (2014) 518-521. https://doi.org/10.1038/nature13433.

[10] A.S. Burrows \& J.P. Ostriker, Astronomical reach of fundamental physics, PNAS, 111, $7 \quad$ (2014) 2409-2416. https://doi.org/10.1073/pnas.1318003111.

[11] D. d'Enterria, P.Z. Skands, S. Alekhin, et al, High-precision $\alpha_{S}$ measurements from LHC to FCC-ee, CERN-PH-TH (2015) 299.

[12] A. Bodek, Precision measurements of electroweak parameters with $\mathrm{Z}$ bosons at the Tevatron, The European Physical JournalC, 76, 3 (2016) 1-12. In Proceedings of the Third LHCP15, 2015. Saint Petersburg, Russia.

[13] M. Thomson, Modern Particle Physics, Cambridge University Press, Cambridge, UK, 2013, 298.

[14] K.A. Olive, et al, (Particle Data Group) The review of particle physics, Chinese Physics C, 38 (2014) \& update (2015) 090001

[15] K. Sundermeyer, Symmetries in Fundamental Physics, Springer, New York, 2014. https://doi.org/10.1007/978-94-007-7642-5.

[16] S.P. Sirag, ADEX Theory: How the ADE Coxeter Graphs Unify Mathematics and Physics, World Scientific Publishing, River Edge, NJ, 2016, pp.2-4. https://doi.org/10.1142/9502.

[17] K.P. Jungmann, Fundamental symmetries and interactions, Nuclear $\begin{array}{lllll}\text { Physics } & A, & 751 & \text { (2005) }\end{array}$ https://doi.org/10.1016/j.nuclphysa.2005.02.099.

[18] C. Quigg, Electroweak symmetry breaking in historical perspective, Annual Review of Nuclear and Particle Science, 65, 1 (2015) 25-42. https://doi.org/10.1146/annurev-nucl-102313-025537.

[19] T. Gannon, Moonshine beyond the Monster: The Bridge Connecting Algebra, Modular Forms and Physics, Cambridge University Press, Cambridge, UK, 2006. https://doi.org/10.1017/CBO9780511535116.

[20] J.F.R. Duncan, M.J. Griffin \& K. Ono, Moonshine, Research in the Mathematical Sciences, 2 (2015) 11. https://doi.org/10.1186/s40687-015-0029-6.

[21] N.J.A. Sloane, Coefficients of modular function $j$ as power series in $q$, The On-Line Encyclopedia of Integer Sequences, (2001) OEIS:A000521.

[22] D. Grumiller, R. McNees, \& J. Salzer, Black holes and thermodynamics, Quantum Aspects of Black Holes, Springer, New York, 2015, pp.27-70.

[23] G.W. Adamson, Convolution square root of A000521, The On-Line Encyclopedia of Integer Sequences, (2009) OEIS:A161361.

[24] T. Eguchi, H. Ooguri \& Y. Tachikawa, Notes on the $K 3$ surface and the Mathieu group $M_{24}$, Experimental Mathematics, 20, 1 (2011) 91-96. https://doi.org/10.1080/10586458.2011.544585.

[25] P.P. Dechant, Clifford algebra is the natural framework for root systems and Coxeter groups. Group theory: Coxeter, conformal and modular groups, Advances in Applied Clifford Algebras, (2015) 115.

[26] H.F. Verheyen, The icosahedral design of the great pyramid, Fivefold Symmetry, World Scientific Publishing, River Edge, NJ, 1992, pp.333-360. https://doi.org/10.1142/97898144394970020.

[27] J. Michell, the New View over Atlantis, Thames \& Hudson, New York, 1995, p.149.

[28] M.A. Sherbon, Wolfgang Pauli and the fine-structure constant, Journal of Science, 2, 3 (2012) 148-154.

[29] R.G. Newton, Galileo's Pendulum: From the Rhythm of Time to the Making of Matter, Harvard University Press, Cambridge, MA, 2004, p.137. https://doi.org/10.4159/9780674041486.

[30] F. Petrie, Origin of the time pendulum, Nature, 132, 3324 (1933) 102.

[31] J. Maldacena, The symmetry and simplicity of the laws of physics and the Higgs boson, European Journal of Physics, 37, 1, 12 (2015) 015802 . 